\title{
Evaluación del debido proceso como factor determinante de la legitimidad de la regulación contable colombiana: una aproximación
}

Carlos Andrés Rodríguez Ramírez ${ }^{1}$

Recibido: 17 de julio de 2016

Aprobado: 19 de octubre de 2016

\begin{abstract}
Rodríguez, C. (2016). Evaluación del debido proceso como factor determinante de la legitimidad de la regulación contable colombiana: una aproximación. Activos, 27, 79-100.
\end{abstract}

Clasificación JEL: G28, G38.

\section{Resumen}

El presente documento propone un análisis de la legitimidad de la regulación contable en Colombia, a partir del concepto de "debido proceso", usando las categorías analíticas propuestas por Suchman y otros autores sobre legitimidad.

El documento concluye que existe un déficit de legitimidad en el ordenamiento regulatorio contable en Colombia, en la medida en que los reguladores mismos incumplen el debido procesos en la emisión de normas de contabilidad, en el marco de la convergencia decretada por la Ley 1314 de 2009, con consecuencias sobre la capacidad del ente regulador -Consejo

1 Contador público egresado de la Universidad Nacional de Colombia, estudiante de la Maestría en Contabilidad de la Universidad Nacional de Colombia. Correo electrónico: carodriguez@unal.edu.co 
Técnico de la Contaduría Pública- y la calidad de la regulación propiamente dicha.

\section{Palabras clave}

Legitimidad, regulación contable, Normas Internacionales de Información Financiera, debido proceso.

Rodríguez, C. (2016). Evaluation of due process like determinant factor of legitimacy of colombian account regulation: An approximation. Activos, 27, 79-100.

\section{Abstract}

This present paper propose an analysis of accounting regulation legitimacy in Colombia, from due process concept, and using the analitic categories proposed by Suchman and other authors about legitimacy concept.

The paper concludes that exist a lack of legitimacy in accounting regulatory orden in Colombia, insofar as regulators fail to comply the due process in the emission process of accounting rules, in the context of convergence enacted for 1314/2009 Act, with consequences on capacity of regulatory body Public Accounting Technical Council- and the quality of regulation itself.

\section{Keywords}

Legitimacy, account regulation, International Financial Reporting Standards, due process.

Rodríguez, C. (2016). Évaluation d'une procédure régulière en tant que déterminant de la légitimité de la réglementation comptable colombienne: une approche. Activos, 27, 79-100.

Cet article propose une analyse de la légitimité de la réglementation comptable en Colombie, du concept de " procédure régulière », en utilisant les 
catégories analytiques proposées par Suchman et d'autres auteurs de la légitimité.

Le document conclut qu'il ya un déficit de légitimité dans le système de réglementation comptable en Colombie, dans la mesure où les régulateurs eux-mêmes violent une procédure régulière dans lémission des normes comptables dans le contexte de la convergence par la loi adoptée 13142009 avec des répercussions sur la capacité du régulateur technique -Conseil de publication comptable et la qualité de la réglementation elle-même.

\section{Mots-clés}

Légitimité, règles comptables, les Normes internationales d'information financière d'une procédure régulière.

\section{Introducción}

Con la expedición de la Ley 1314 de 2009, se da inicio formal en Colombia al proceso de convergencia de las normas de contabilidad, información financiera y aseguramiento de la información, hacia "estándares internacionales de aceptación mundial acordes con las mejores prácticas y con la rápida evolución de los negocios" (términos usados en la Ley 1314 de 2009 pero que son cuestionados por García y Sierra, 2012).

Con relación a las normas de información financiera, el Consejo Técnico de la Contaduría Pública - CTCP ha sugerido a los Ministerios de Hacienda y Crédito Público y de Comercio, Industria y Turismo la adopción de los estándares emitidos por el IASB, tanto las Normas Internacionales de Información Financiera - NIIF (en inglés International Financial Reporting Standards - IFRS) como las Normas Internacionales de Información Financiera para pequeñas y medianas entidades (NIIF para Pymes; IFRS para Pymes), así como el desarrollo de una regulación especial para las entidades consideradas como microempresas (contabilidad simplificada). 
Estas sugerencias fueron aceptadas por dichas entidades, y como resultado se publicaron los Decretos 2784 de 2012, 2706 de 2012 y 3022 de 2013, a través de los cuales se expiden los marcos normativos aplicables a las entidades del sector privado colombiano. No obstante, algunos autores consideran que la adopción de dichos estándares no es pertinente (ver por ejemplo Álvarez y Gómez, 2008; García y Sierra, 2012; Ferrer, 2013). Estas afirmaciones parecen tener sentido cuando se observan las modificaciones que han tenido que realizarse a la regulación contable financiera inicialmente emitida, ante las presiones ejercidas por algunos interesados que consideran que dichas reformas afectan abiertamente sus resultados financieros.

El presente documento considera que esta problemática deriva de una legitimidad inadecuada de la regulación contable inicialmente expedida en el marco de la Ley 1314, la cual a su vez deriva de fallas en el debido proceso que deben realizar el normalizador y los reguladores de los marcos normativos contables aplicables a las entidades del sector privado.

Con el fin de desarrollar esta idea el documento abordará, en primer lugar, los aspectos claves de la teoría de la legitimidad discutiendo sobre los tipos de legitimidad que pueden perseguir las organizaciones, para identificar con posterioridad cuál de ellos sería potencialmente aplicable al modelo de emisión de regulación del Consejo Técnico de la Contaduría Pública - CTCP como organismo de normalización dentro de la regulación contable financiera colombiana. En un tercer momento se realiza un recuento del proceso seguido en Colombia para la emisión de la nueva regulación y se identifican los ajustes posteriores que el Gobierno Nacional ha tenido que hacer a dicha regulación analizando si estos podían ser advertidos antes de la emisión inicial de los decretos que reglamentaron la nueva regulación contable financiera. Finalmente, se presentan las conclusiones del trabajo y el posible impacto que dichas líneas de acción tendrían para el trabajo que debe adelantar el CTCP como regulador. 


\section{Teoría de la legitimidad}

Dado que este artículo aborda las modificaciones a los nuevos marcos normativos de información financiera en Colombia como un problema de legitimidad de la regulación inicialmente emitida, la teoría de la legitimidad cobra importancia. En este sentido es necesario realizar un análisis básico sobre las teorías que explican la legitimidad, se pueden identificar dos corrientes de estudio: la estratégica y la institucional.

Desde la perspectiva estratégica, la legitimidad es concebida como un recurso operacional que las organizaciones extractan de la cultura en la cual están inmersas y que emplean para alcanzar sus objetivos. En consecuencia, se considera que la legitimidad puede ser administrada por medio de distintos medios a través de los cuales las organizaciones manipulan y crean simbolismos que están orientados a la acumulación de aceptación social.

Por su parte, la perspectiva institucional considera que las dinámicas de los distintos sectores generan presiones culturales sobre la legitimidad de las organizaciones y que dichas presiones están más allá del control de las organizaciones. En consecuencia, las definiciones culturales son las que determinan la constitución y estructura de las organizaciones, su funcionamiento y la forma como deben ser entendidas y evaluadas (Bamber y McMeeking, 2016; Suchman, 1995).

Teniendo en cuenta que en las organizaciones del mundo real coexisten presiones institucionales que dan forma a las organizaciones y cambios operacionales estratégicos realizados por las organizaciones para administrar la legitimidad, Suchman (1995) considera importante incorporar las dos corrientes anteriormente mencionadas en el análisis de la legitimidad de las organizaciones.

En este sentido, Suchman (1995) afirma que:

La legitimidad es una percepción o asunción generalizada de que las acciones de una entidad son deseables, propias, o apropiadas dentro de 
algún sistema socialmente construido de normas, valores, creencias y definiciones (p. 574).

Por ende, la legitimidad puede desearse en procura de la continuidad, la credibilidad, el soporte activo o el soporte pasivo, de una organización. El propósito perseguido dependerá del contexto en el cual se desenvuelva la organización y las características propias de la misma tales como la antigüedad, la especificidad técnica y su estructura orgánica, entre otros.

Los medios a través de los cuales las organizaciones pueden hacer que sus actividades sean consideradas como deseables, propias o apropiadas dentro de un sistema socialmente construido de normas, valores, creencias y definiciones pueden ser muy variados. No obstante, Suchman (1995) distingue tres grandes tipos de legitimidad, a saber, la legitimidad pragmática, la legitimidad moral y la legitimidad cognitiva, cada una de estas con distintos objetivos, grados de dificultad y dinámicas comportamentales. Es de resaltar que estos tipos de legitimidad no son mutuamente excluyentes sino que pueden coexistir bajo distintas estrategias dentro de una misma organización.

\section{Tipologías de la legitimidad}

\section{Legitimidad pragmática}

La legitimidad pragmática, parte del supuesto de que la audiencia considera como legitimas a aquellas organizaciones que realizan actividades que son favorables para sus propios intereses y que no considera legitimas a aquellas organizaciones que realizan actividades que les perjudican. En consecuencia, se generan intercambios directos entre la organización y la audiencia generando interdependencias políticas, económicas o sociales a través de las cuales las acciones de la organización afectan el bienestar de la audiencia.

Dado el interés que tiene la audiencia en conocer cómo las acciones organizacionales impactan sus intereses personales, a menudo la audiencia es considerada como un constituyente de dichas acciones, puesto que es 
ella quien a través del escrutinio del comportamiento organizacional y la continua evaluación y aprobación o desaprobación de las políticas organizacionales, contribuye o entorpece la permanencia de las organizaciones.

$\mathrm{Al}$ interior de este tipo de legitimidad, Suchman (1995) identifica tres clases de legitimidad, a saber:

a. La legitimidad por intercambio, en la cual los constituyentes soportan a la organización por los beneficios directamente recibidos. Este tipo de legitimidad se consigue generalmente con la incorporación de políticas organizacionales que tienen en cuenta la evaluación esperada que los constituyentes harán de las actividades realizadas por la organización.

b. La legitimidad influenciada, en la cual los constituyentes soportan a la organización no por los beneficios directamente recibidos sino por los beneficios mayores que se obtendrán de las actividades realizadas por la organización. Este tipo de legitimidad se consigue generalmente a través de la incorporación de los constituyentes en la estructura de definición de políticas organizacionales, o adoptando los estándares de mejora de los constituyentes como suyos propios.

c. La legitimidad de disposición, se basa principalmente en la personificación de las organizaciones con lo cual los constituyentes soportan a la organización por las características que posee la misma como individuo, es decir, sus objetivos, gustos, estilos y personalidades. Bajo esta perspectiva las organizaciones son consideradas como actores autónomos, coherentes y moralmente responsables.

\section{Legitimidad moral}

La legitimidad moral refleja, en palabras de Suchman (1995), "una evaluación positiva normativa de la organización y de sus actividades" (p. 579), este tipo de legitimidad se enfoca en si las actividades realizadas por la organización son las correctas y no en si las actividades realizadas benefician 
a los constituyentes. En consecuencia, las actividades organizacionales son juzgadas desde el bienestar social y no desde el individual. Al interior de este tipo de legitimidad se pueden distinguir cuatro corrientes:

a. La legitimidad consecuencial: las organizaciones deberían ser evaluadas por sus logros o sus productos. No obstante, este enfoque presenta inconvenientes para la evaluación de algunos productos que son inherentemente difíciles de medir.

b. Le legitimidad procedimental: las organizaciones pueden ganar legitimidad mediante la adopción de técnicas y procedimientos socialmente aceptados. Este tipo de legitimidad cobra importancia cuando los productos generados por la organización no son fácilmente medibles.

c. La legitimidad estructural: el soporte es dado a las organizaciones cuya estructura organizacional es considerada moralmente favorable.

d. La legitimidad personal: la legitimidad yace en el carisma de los líderes organizacionales.

\section{Legitimidad cognitiva}

Por último, la legitimidad cognitiva es la mejor de todas, pero al mismo tiempo, la más difícil de alcanzar. Suchman (1995) distingue dos variantes que deben considerarse. Primero, la comprensibilidad que consiste en hacer que las actividades de la organización sean entendidas e interiorizadas a través del engranaje de estas con los sistemas de creencias instaurados y con las experiencias de la vida diaria de la audiencia. Segundo, que las actividades de la organización sean percibidas como dadas por sentado, es decir, que no son objeto de discusión. Este tipo de legitimidad es considerado el más sutil y a la vez el más poderoso, no obstante, este tipo de legitimidad escapa del control de las organizaciones. 


\section{La legitimidad en el marco de la regulación contable en Colombia}

Considerando que 1) la regulación contable colombiana, tal como lo exige la Ley 1314 de 2009, debe tener en cuenta la conveniencia nacional de adoptar estándares internacionales y no solo el bien particular de algunos constituyentes y 2) la regulación contable como producto no es fácilmente medible para la determinación de si es o no conveniente para el país, este articulo propone evaluar la legitimidad de la regulación inicialmente emitida por el CTCP a través del debido procedimiento que se siguió para su expedición. De acuerdo con la categorización realizada por Suchman (1995) presentada en el aparte anterior, se pretende hacer una aproximación a la evaluación de la legitimidad moral-procedimental de la regulación contable financiera colombiana.

\section{El debido proceso para la emisión de la nueva regulación contable financiera en Colombia}

Con la expedición de la Ley 1314/2009 se da inicio a un nuevo proceso de regulación contable de las entidades privadas en el marco de un proceso de convergencia de las normas de contabilidad, de información financiera y de aseguramiento de la información hacia estándares internacionales de aceptación mundial. Lo anterior con el objetivo de "apoyar la internacionalización de las relaciones económicas y mejorar la productividad, la competitividad y el desarrollo armónico de la actividad empresarial" (Congreso de la República de Colombia, 2009).

En el artículo 6 de la Ley 1314, se establece que los Ministerios de Hacienda y Crédito Público y de Comercio, Industria y Turismo, obrando conjuntamente, serán los encargados de expedir los nuevos marcos normativos aplicables a las entidades privadas, y que el CTCP será el organismo de normalización técnica de las normas. 
Los artículos 7 y 8 de la citada Ley señalan los elementos que determinan el debido proceso que deben seguir los organismos de regulación y el organismo de normalización técnica para la emisión de la nueva regulación. En el caso de los Ministerios de Hacienda y Crédito Público y de Comercio, Industria y Turismo, la ley les exige:

1) verificar que el proceso de elaboración de los proyectos por parte del CTCP haya sido abierto, transparente y de público conocimiento; 2) considerar las observaciones y recomendaciones realizadas por la DIAN, por los organismos responsables del diseño y manejo de la política económica y por las entidades estatales que ejercen funciones de inspección, vigilancia o control;3) tener en cuenta los comentarios realizados durante la etapa de exposición pública de los proyectos así como el análisis que haga el CTCP a los mismos sobre su pertinencia; 4) publicar las normas junto con los fundamentos de sus conclusiones en medios que garanticen su amplia divulgación; 5) revisar la consistencia de la regulación emitida; y 6) las demás que determine el Gobierno Nacional para garantizar buenas prácticas y un debido proceso en la expedición de la regulación (Ley 1314).

Asimismo, el artículo 8 de la Ley 1314, india que el CTCP tendrá que:

1) enviar a los Ministerios cada seis meses un programa de trabajo; 2) asegurar que las propuestas se ajusten a las mejores prácticas internacionales; 3 ) utilizar procedimientos que sean ágiles, flexibles, transparentes y de público conocimiento; 4) tener en cuenta, en la medida de lo posible, la relación costo-beneficio de las normas proyectadas; 5) tomar como referente para la elaboración de las propuestas los estándares más recientes y de mayor aceptación que hayan sido expedidos o estén próximos a ser expedidos por los organismos internacionales reconocidos a nivel mundial como emisores de estándares internacionales; 6) evaluar si los estándares utilizados como referente resultan eficaces o apropiados para los entes en Colombia y comunicar su evaluación a los Ministerios para que estos decidan sobre su conveniencia e implicaciones de acuerdo con el interés público y el bien común; 7) tener en cuenta para las propuestas de norma las diferencias entre los entes económicos, en razón a su tamaño, forma de organización 
jurídica, el sector al que pertenecen, su número de empleados y el interés público involucrado en su actividad, para que los requisitos y obligaciones que se establezcan resulten razonables y acordes a tales circunstancias; 8) propender por la participación voluntaria de reconocidos expertos en la materia; 9) establecer comités técnicos ad honórem conformados por las autoridades, preparadores, aseguradores y usuarios de la información financiera; 10) considerar las observaciones y recomendaciones realizadas por la DIAN, por los organismos responsables del diseño y manejo de la política económica, por las entidades estatales que ejercen funciones de inspección, vigilancia o control, y por quienes participen en los procesos de discusión pública; 11) publicar, para su discusión pública, los borradores de proyectos en medios que garanticen su amplia divulgación; 12) publicar los proyectos definitivos al tiempo que estos sean enviados a los Ministerios; 13) velar porque sus decisiones sean adoptadas en tiempos razonables y con las menores cargas posibles para sus destinatarios; y 14) promover, en coordinación con otras entidades, un proceso de divulgación, conocimiento y comprensión que busque desarrollar actividades tendientes a sensibilizar y socializar en las empresas del país y demás interesados, los procesos de convergencia hacia estándares internacionales.

En cumplimiento del mandato de la Ley 1314, el 22 de diciembre de 2010, el CTCP publicó para discusión de los interesados el documento Propuesta para el direccionamiento estratégico del Consejo Técnico de la Contaduría Pública (CTCP) y el entendimiento común del proceso de convergencia de las normas de contabilidad, de información financiera y de aseguramiento de la información, con estándares internacionales, el cual estuvo dispuesto para comentarios hasta el 30 de enero de 2011.

Este documento contenía la propuesta de la ruta a seguir para dar cumplimiento a lo exigido en la Ley 1314, proponía dividir las entidades privadas en cinco grupos para discriminar los marcos reguladores aplicables:

1) entidades que emiten títulos de deuda y/o de patrimonio en mercados públicos y las entidades de interés público, 2) los inversionistas extranjeros que no hagan parte del grupo 1 y las empresas de tamaño grande y 
mediano, 3) pequeñas empresas nacionales, 4) micro empresas nacionales y 5) entidades sin ánimo de lucro.

En cuanto a regulación contable financiera, el CTCP (2010) proponía que el primer grupo aplicara NIIF (IFRS por sus siglas en inglés) y el segundo grupo aplicara las NIIF para Pymes (IFRS for SME por sus siglas en inglés). Por su parte para los grupos 3,4 y 5 se propuso la creación de una regulación local especial que se ciñera a los criterios establecidos en la Ley 1314 de 2009.

El CTCP recibió cuarenta y ocho comentarios al documento publicado el 22 de diciembre, a partir de estos, modificó la propuesta inicial la cual publicó en su versión final el 22 de junio de 2011 bajo el título Direccionamiento estratégico del proceso de convergencia de las normas de contabilidad e información financiera y de aseguramiento de la información, con estándares internacionales.

López y Lourido (2011) consideran que estos cuarenta y ocho comentarios recibidos sobre la propuesta presentada por el CTCP representan una participación muy baja de la comunidad académica, profesional y empresarial. No existe un diagnóstico del por qué se presenta este bajo nivel de participación, pero en todo caso este es un primer momento en el que el proceso comienza a presentar fracturas de legitimidad.

Finamente el documento final emitido por el CTCP propuso la conformación de tres grupos de entidades (empresas): grupo 1 para entidades que cotizan en el mercado de valores o que son consideradas de interés público; grupo 2 para las empresas grandes, medianas y pequeñas que no pueden ser clasificadas en el grupo 1; grupo 3 para las microempresas.

Posteriormente, el 15 de diciembre de 2011, el CTCP publicó para comentarios el documento denominado Propuesta de modificación a la conformación de los grupos de entidades para la aplicación de NIIF (IFRS). En este documento se proponía una modificación en la conformación de los grupos definidos en el direccionamiento estratégico del 22 de junio de 
2011. Sobre este documento se recibieron 24 comentarios, un número que vale la pena resaltar, porque es aún más bajo que el número de comentarios recibidos sobre el documento inicial de direccionamiento, teniendo en cuenta el universo de entidades que son afectadas por dicha regulación.

Con base al documento emitido del 15 de diciembre de 2011 y los comentarios recibidos al mismo, el CTCP publicó el 5 de diciembre de 2012 la versión final del documento Direccionamiento estratégico del proceso de convergencia de las normas de contabilidad e información financiera y de aseguramiento de la información, con estándares internacionales. En esta versión se incorporaron los cambios puestos a discusión el 15 de diciembre quedando las entidades clasificadas en tres grupos definitivos, los cuales no sufrieron mayor modificación.

Para el caso de las entidades que quedaron clasificadas en el grupo tres (micro y pequeñas empresas), el 21 de diciembre de 2011 el CTCP publicó para comentarios del público el documento Proyecto de norma de información financiera para las microempresas, el cual fue puesto a discusión hasta el 31 de marzo de 2012. Dicha propuesta proponía básicamente una contabilidad simplificada basada principalmente en el costo histórico de las transacciones y unos estados financieros básicos (estado de situación financiera, estado de resultados y notas explicativas).

Fruto de la exposición del documento a discusión de los interesados se recibieron 21 comentarios y se presentó a los Ministerios de Hacienda y Crédito Público y de Comercio, Industria y Turismo la propuesta definitiva. Esta propuesta se convertiría en el Decreto Nacional 2706 de 2012, mediante el cual se obliga a que las entidades que cumplan la definición de microempresa contenida en el anexo del decreto, apliquen un marco técnico contable simplificado basado esencialmente en el costo histórico. Dicho decreto adicionalmente fijó para estas entidades el cronograma de transición que deberían aplicar definiendo en esencia tres periodos, a saber: periodo de preparación obligatoria (año 2013), periodo de transición (año 2014) y primer periodo de aplicación del nuevo marco normativo (año 2015). 
Para el grupo dos, el 23 de octubre de 2012 el CTCP puso a discusión el documento de Propuesta de norma internacional de información financiera para Pymes (IFRS for Smés) para la convergencia hacia estándares internacionales, el cual estuvo dispuesto para comentarios de los interesados hasta el 31 de marzo de 2013. En dicho documento, en línea con el direccionamiento estratégico final, se proponía que las entidades categorizadas en el grupo 2 aplicaran las NIIF para Pymes emitidas por el IASB sin modificación alguna, es decir, que se hiciera una adopción plena de dichos estándares. Durante el periodo de exposición para comentarios de los interesados se recibieron 13 comentarios dentro de los cuales se encontraban tres universidades, cuatro superintendencias, la DIAN, el Banco de la República, la Asociación Nacional de Fondos de Empleados (ANALFE) y solo tres eran participantes independientes.

Surtido el proceso de exposición a comentarios del público, el 1 de octubre de 2013, el CTCP presentó a los Ministerios de Hacienda y Crédito Público y de Comercio, Industria y Turismo la sustentación de la propuesta sobre la aplicación de las NIIF para PYMES. Como resultado de lo anterior, los ministerios expiden el Decreto 3022 del 27 de diciembre de 2013, a través del cual se obliga a las entidades clasificadas en el grupo 2 a aplicar las NIIF para Pymes emitidas por el IASB. Adicionalmente, el decreto determina el cronograma para la transición hacia el nuevo marco normativo estableciendo tres periodos, a saber: el periodo de preparación obligatoria (año 2014), periodo de transición (año 2015) y primer periodo de aplicación del nuevo marco normativo (año 2016).

Finalmente, con relación a las entidades agrupadas en el grupo 1, el 21 de diciembre de 2011, el CTCP puso a discusión del público el documento denominado Propuesta de normas de contabilidad e información financiera para la convergencia hacia estándares internacionales, el cual estuvo dispuesto para comentarios de los interesados hasta el 31 de marzo de 2012. En esta propuesta el CTCP proponía que las entidades clasificadas dentro de este grupo aplicaran las NIIF emitidas por el IASB sin ninguna modificación, en otras palabras, que se hiciera una adopción plena de las NIIF. 
Este documento recibió cincuenta y siete comentarios, una cifra que está en el promedio de la participación del modelo de regulación concertada emprendido por el CTCP. Surtido el proceso de exposición para comentarios se presentó a los Ministerios el 16 de octubre de 2012 la propuesta de regulación contable financiera para el grupo uno.

Fruto de esta propuesta, el 28 de diciembre de 2012, los Ministerios de Hacienda y Crédito Público y de Comercio, Industria y Turismo expidieron el Decreto 2784, en el cual se exigía a las entidades clasificadas en el grupo 1 la aplicación de las NIIF sin ninguna modificación y se establecía el siguiente cronograma de transición al nuevo marco de regulación: periodo de preparación obligatoria (año 2013), periodo de transición (año 2014) y primer periodo de aplicación del nuevo marco normativo (año 2015).

\section{Las excepciones en los marcos regulatorios derivados de la Ley 1314}

El 29 de agosto de 2013, a menos de un año de expedición del marco normativo de información financiera para las entidades clasificadas en el grupo 1, se realiza la primera excepción a la regulación definida en el Decreto 2784 de 2012 a través del Decreto 1851 de 2013. Esta excepción consistía en que los establecimientos bancarios, corporaciones financieras, compañías de financiamiento, cooperativas financieras, organismos cooperativos de grado superior y entidades aseguradoras no aplicaran en sus estados financieros individuales la NIC 39 ni la NIIF 9, respecto del tratamiento de la cartera de crédito y su deterioro, y que tampoco aplicaran la NIIF 4 respecto del tratamiento de las reservas técnicas catastróficas para el ramo de terremoto y la reserva de desviación de siniestralidad para el ramo de seguros de riesgos laborales.

Es de notar que a pesar de que el marco normativo contó con tiempos para su revisión y manifestaciones de ajustes, los actores interesados (afectados) hicieron posteriormente lobby frente a los organismos ministeriales reguladores para generar excepciones a las normas expedidas, esto muestra 
o bien que no hubo conocimiento del proceso de construcción normativa por parte del CTCP entre los actores interesados, o bien que las entidades afectadas nunca tuvieron en mente las afectaciones que los nuevos modelos de regulación traerían para el normal desarrollo de sus negocios.

Adicionalmente, el Decreto 2267 del 11 de noviembre de 2014, amplió las excepciones introducidas por el Decreto 1851 de 2013 con relación a los estados individuales de las entidades clasificadas en el grupo 1. En consecuencia, la excepción finalmente establece que los establecimientos bancarios, corporaciones financieras, compañías de financiamiento, cooperativas financieras, organismos cooperativos de grado superior y entidades aseguradoras no aplicarán en sus estados financieros individuales la NIC 39 y la NIIF 9 respecto al tratamiento de la cartera de crédito y su deterioro, así como a la clasificación y la valoración de inversiones, y que tampoco se aplicará la NIIF 4 en lo relacionado con las reservas técnicas catastróficas para el ramo de terremoto, las reservas de desviación de siniestralidad y la reserva de insuficiencia de activos.

Dichas excepciones cobran importancia cuando se observa que dentro de los comentarios allegados por la Superintendencia Financiera de Colombia al CTCP, se afirmaba que era necesario hacer el estudio de los posibles impactos que las NIIF podrían tener en las entidades del sector financiero, y se resaltaba que aproximadamente solo el $28 \%$ de las entidades del sector a nivel mundial han aplicado dichos estándares. Adicionalmente, la Superintendencia presentaba las justificaciones que habían llevado a que en los países europeos no se hubiera hecho la adopción de la NIC 39 y la NIIF 9, sumado a ello también era cuestionado el cronograma planteado para la adopción de las NIIF en Colombia.

A pesar de los comentarios presentados por la Superintendencia, la propuesta que presentó el CTCP a los Ministerios de Hacienda y Crédito Público y de Comercio, Industria y Turismo no expuso la necesidad de establecer alguna excepción en la aplicación de las NIIF. Esto pone en evidencia un incumplimiento al debido proceso exigido por la Ley 1314 de 2009, pues muy a pesar de las advertencias señaladas realizadas por la 
Superintendencia Financiera, el CTCP prefirió una vía expedita de regulación sin tener en cuenta las observaciones y sin allegar los potenciales estudios de impacto normativo sobre diferentes sectores de la economía.

Pero estas no fueron las únicas excepciones, el 23 de diciembre de 2015, se expidió el Decreto 2496 por medio del cual se modifica el Decreto 2420 de 2015 Único Reglamentario de las Normas de Contabilidad, de Información Financiera y de Aseguramiento de la Información y se dictan otras disposiciones en el cual se hacen excepciones a las entidades pertenecientes a los sectores salud y cooperativo.

La primera excepción (artículo 2 del Decreto 2496) hace una modificación al cronograma de aplicación del nuevo marco normativo para las entidades clasificadas dentro del grupo 2 que conforman el Sistema General de Seguridad Social en Salud y para las Cajas de Compensación Familiar. Aplaza por un año las actividades del cronograma definido en el Decreto 3022 de 2013 quedando, en consecuencia, el cronograma para estas entidades de la siguiente manera: el periodo de preparación obligatoria (año 2015), periodo de transición (año 2016) y primer periodo de aplicación del nuevo marco normativo (año 2017).

Dicha excepción cobra mayor importancia en tanto que revisando los comentarios de quienes opinaron sobre la propuesta de regulación para el grupo 2 puesta a consideración del público, se observa que dos participantes independientes y una Superintendencia cuestionan si los plazos propuestos para la aplicación de las NIIF para Pymes eran adecuados. A lo anterior, pueden sumarse las participaciones que algunos interesados realizaron en otros documentos publicados por el CTCP, en los cuales se cuestionaba la pertinencia de los plazos sugeridos por este organismo de normalización.

En consecuencia, con esta excepción se pone en evidencia que algunas entidades, e incluso un sector entero, no estaban preparados para la implementación de las NIIF para Pymes, y que el CTCP pasó por alto los comentarios que lo advertían desde sus inicios, incumpliendo así con el debido proceso establecido en el artículo 8 de la Ley 1314 de 2009. 
La segunda excepción -contenida en el artículo 3 del Decreto 2496 de 2015- es aplicable a las entidades vigiladas por la Superintendencia de la Economía Solidaria con independencia del grupo al cual pertenezcan. La excepción consiste en que los aportes de estas entidades no sean considerados como un pasivo, tal como lo exige las normas internacionales (tanto NIIF como NIIF para Pymes), sino que sigan siendo clasificadas como parte del patrimonio de dichas entidades.

En este caso se evidencia que la participación que tuvieron tanto la Superintendencia de la Economía Solidaria, como algunas cooperativas que participaron con comentarios en las propuestas normativas que exponía el CTCP para los grupos 1 y 2 , exponían la inconveniencia de aplicar a este tipo de entidades los criterios establecidos por las NIIF y las NIIF para Pymes. Adicionalmente, suministraban información sobre el problema causado por la valoración bajo NIIF sufrido por entidades pertenecientes a la economía solidaria, y en general a las entidades sin ánimo de lucro en otros países que habían adoptado NIIF y sobre las excepciones que se realizaron para este tipo de entidades.

No obstante, la propuesta del CTCP no contemplaba excepciones para las entidades del sector solidario y estas nunca se contemplaron en el proceso de discusión ni de emisión normativa.

\section{Conclusiones y recomendaciones}

En el documento se parte de la teoría de la legitimidad y los tipos de legitimidad identificados por Suchman (1995), y se identificó la necesidad de evaluar la regulación contable financiera a partir de su legitimidad moral-procedimental. Posteriormente, se presentó un detalle sobre los componentes del debido proceso seguido en Colombia para la expedición inicial de la regulación contable financiera aplicable a las entidades privadas y las excepciones que a posterioridad se realizaron. 
De todo lo anterior, se concluye que, si bien la Ley 1314 de 2009 exige un debido proceso para la emisión de la regulación contable financiera en convergencia con estándares internacionales, el CTCP no lo siguió de una adecuada manera. Los ajustes posteriores realizados a la regulación inicialmente emitida evidenciando la incorrecta evaluación que el CTCP está haciendo de los comentarios realizados por los participantes, los cuales posteriormente están derivando en excepciones que hacen cuestionar la legitimidad de la regulación expedida. Y no solo eso, pone en cuestión la legitimidad del mismo proceso de regulación, al ignorar los comentarios y observaciones sustentadas de las entidades reguladas, le resta capacidad y sentido al proceso y deja al CTCP como una entidad donde los actores no tienen cabida, además que deja en duda la verdadera capacidad técnica del CTCP y las motivaciones de la regulación emitida.

El CTCP debe implementar un debido proceso mucho más completo, participativo y transparente para que de esta forma se garantice la legitimidad de la regulación expedida y, en consecuencia, su permanencia en el tiempo. Esta recomendación cobra importancia en la medida en que tal como firman Meyer y Rowan (1991):

Las organizaciones que pierden legitimidad en la aceptación de la responsabilidad de sus actividades son más vulnerables a ser categorizadas como negligentes, irracionales o innecesarias (p. 75).

Es decir, que de no ajustarse el debido proceso que garantiza una legitimidad instrumental al proceso de regulación, puede invalidarse la capacidad del Consejo Técnico como ente regulador técnico y desviar el peso institucional de la emisión normativa hacia otras entidades del Estado donde los regulados tienen mayor "capacidad de incidencia", en este caso los Ministerios de Hacienda, de Industria y Comercio o las Superintendencias Sectoriales, con lo cual se rompería definitivamente la institucionalidad establecida por la ley 1314 y potencialmente se presentaría una mayor dispersión de la regulación contable local. 


\section{Referencias}

Álvarez, H., y Gómez, M. (2008). Adopción de la normativa contable internacional: ¿es el camino correcto? Análisis del Proyecto de Ley 165 del 2007. Revista internacional Legis de Contabilidad y Auditoría, 35, (pp. 99-126).

Bamber, M., \& McMeeking, K. (2016). An examination of international accounting standard-setting due process and the implications for legitimacy. The British Accounting Review, 48(1), 59-73.

Congreso de la República de Colombia. (13 de julio de 2009). Por la cual se regulan los principios y normas de contabilidad e información financiera y de aseguramiento de información aceptados en Colombia, se señalan las autoridades competentes, el procedimiento para su expedición y se determinan las entidades responsables de vigilar su cumplimiento. [Ley 1314 de 2009]. Diario Oficial: 47.409.

CTCP. (2010). Propuesta para el direccionamiento estratégico del Consejo Técnico de la Contaduría Pública (CTCP) y el entendimiento común del proceso de convergencia de las normas de contabilidad, de información financiera y de aseguramiento de la información. Documento disponible en la dirección http:// www.ctcp.gov.co/_files/documents/DOC_CTCP_6B2N7_149.pdf

CTCP. (2011a). Direccionamiento estratégico del proceso de convergencia de las normas de contabilidad e información financiera y de aseguramiento de la información, con estándares internacionales. Disponible en la página http://www.mincit.gov.co/publicaciones/17878/consejo_tecnico_de_la_contaduria_publica

CTCP. (2011b). Propuesta de modificación a la conformación de los grupos de entidades para la aplicación de NIIF (IFRS).

CTCP. (2011c). Propuesta de normas de contabilidad e información financiera para la convergencia hacia estándares internacionales.

CTCP. (2011d). Proyecto de norma de información financiera para las microempresas.

CTCP. (2012a). Direccionamiento estratégico del proceso de convergencia de las normas de contabilidad e información financiera y de aseguramiento de la información, con estándares internacionales.

CTCP. (2012b). Documento de sustentación de la propuesta del proyecto de norma de información financiera para las microempresas (grupo 3).

CTCP. (2012c). Propuesta de Norma Internacional de Información Financiera para Pymes (IFRS for Sme's) para la convergencia hacia estándares internacionales. 
CTCP. (2012d). Documento de sustentación de la propuesta a los Ministerios de Hacienda y Crédito Público y de Comercio, Industria y Turismo sobre la aplicación de las Normas Internacionales de Información Financiera en Colombia, grupo 1.

CTCP. (2013). Documento de sustentación de la propuesta a los Ministerios de Hacienda y Crédito Público y de Comercio, Industria y Turismo sobre la aplicación de la Norma Internacional de Información Financiera - NIIF para pequeñas y medianas entidades (Pymes) en Colombia.

Ferrer, A. (2013). Análisis del proceso de convergencia a Normas Internacionales de Contabilidad e Información Financiera desde los factores intrínsecos al sistema contable en Colombia. Cuadernos de contabilidad, 14(36), 971-1007.

García, N., y Sierra, E. (2012). Un análisis de la reforma contable en Colombia. Revista Internacional Legis de Contabilidad y Auditoría, 51, (pp. 39-54).

López, c., y Lourido, F. (2011). Convergencia a estándares internacionales de información financiera y de aseguramiento en Colombia: análisis de documentos de direccionamiento estratégico del CTCP. Revista Internacional Legis de Contabilidad y Auditoría, 97-134.

Meyer, J., \& Rowan, B. (1991). Institutionalized organizations: formal structure as myth and ceremony. American Journal of Sociology, 83(2), 340-363.

Ministerio de Hacienda y Crédito Público, Ministerio de Industria, Comercio y Turismo. (28 de diciembre de 2012a). Decreto 2784 de 2012. Diario Oficial: 48.658.

Ministerio de Hacienda y Crédito Público, Ministerio de Industria, Comercio y Turismo. (27 de diciembre de 2012b). Decreto 2706 de 2012. Diario Oficial: 48.657.

Ministerio de Hacienda y Crédito Público; Ministerio de Industria, Comercio y Turismo. (29 de agosto de 2013a). Decreto 1851 de 2013. Diario Oficial: 48.897.

Ministerio de Hacienda y Crédito Público; Ministerio de Industria, Comercio y Turismo. (27 de diciembre de 2013b). Decreto 3022 de 2013. Documento disponible en la página http://www.mincit.gov.co/loader.php?1Servicio=D ocumentos\&lFuncion $=$ verPdf\&id=4585\&name=Decreto3022_2013_p.pdf

Ministerio de Hacienda y Crédito Público; Ministerio de Industria, Comercio y Turismo. (11 de noviembre de 2014). Decreto 2267 de 2014. Diario Oficial: 49.332 . 
Ministerio de Hacienda y Crédito Público; Ministerio de Industria, Comercio y Turismo. (23 de diciembre de 2015). Decreto 2496 de 2015. Diario Oficial: 49.735 .

Pacter, P. (2005). What exactly is convergence? Int. J. Accounting, Auditing and Performance Evaluation, 2(1-2), 67-83.

Stephen, Z. (2012). La evolución del IASC al IASB, y los retos que enfrenta. Contaduría. Universidad de Antioquia, 60, (pp. 119-164).

Suchman, M. (1995). Managing Legitimacy: Strategic and institutional approaches. The Academy of Management Review, 20(3), 571-610. 